\title{
Time Of Flight Secondary Ion Mass spectrometry: Chemical Imaging
}

Sachin Attavar ${ }^{1}$, David A. Cole ${ }^{1}$, Arwa Ginwalla ${ }^{2}$, and Jim Gibson ${ }^{3}$.

1. Evans Analytical Group, East Windsor-NJ, USA.

2. Evans Analytical Group, Sunnyvale-CA, USA.

3. Evans Analytical Group, Chanhassen-MN, USA.

Time-of-Flight Secondary Ion Mass Spectrometry (TOF-SIMS) is a surface analytical technique that focuses a pulsed beam of primary ions onto a sample surface, producing secondary ions in a sputtering process. Analysis of the secondary ions provides information about the molecular and elemental species present on the surface. Along with the molecular species, TOF-SIMS is a survey technique with ability to detect all elements in the periodic table, including H. TOF-SIMS combines the analytical power of mass spectrometry with the imaging ability of microprobe techniques. The primary ion beam is rastered over the surface, thereby producing secondary ions from various locations on the surface. Selected ion images may be produced by plotting counts from a given mass range or combination of ranges versus the coordinates from which they arose. The spatial resolutions in TOF-SIMS range around 1-3 microns. In certain ideal samples, sub-micron resolution is achievable. In recent advances, development of Argon gas cluster ion beam (GCIB) has given TOF-SIMS a capability of 3-D mapping, keeping the molecular information intact. Argon cluster GCIB is able to remove material layer by layer without affecting the chemical integrity of the layer below.

Three examples have been discussed below to show the capabilities of the TOF-SIMS instrument from three perspectives: medical device, pharmaceutical drugs, and tissue sections. Figure 1 shows the distribution of antibiotics in bone cement ${ }^{1}$. The mixing of antibiotics into bone cement to prevent infection has been used since the 1970's and is still a common practice in total joint replacement surgery. There have been many studies on the elution rate of the antibiotics and the effects of the antibiotics on the properties of bone cement. TOF-SIMS is a direct determination of the distribution of antibiotic (Tobramycin) within bone cement. The TOF-SIMS data indicates that on a smaller scale, Tobramycin is found in 'clumps' ranging from $<1 \mu \mathrm{m}$ to $35 \mu \mathrm{m}$, with a mean size of $1 \mu \mathrm{m}$. This study could aid in developing methods to optimize the distribution of antibiotic in the bone cement to provide the maximum patient benefit and lowest cost treatment.

TOF-SIMS imaging can be used to study a wide range of pharmaceutical problems. A list of possible TOF-SIMS applications includes the investigation of coating homogeneity, particle size analysis and distribution, process analytical control, cleaning validation, root cause investigation, and reverse engineering. The ability of TOF-SIMS for molecular specificity and imaging is demonstrated using a cross-section of over-the-counter lansoprazole tablet is shown in figure 2. Oral pharmaceutical tablets are mixtures of chemical species which include the active pharmaceutical ingredient (API) and organic and inorganic excipients. In Figure 2a, a chemical map of $\mathrm{Mg}$ shows that it present in the shell (Talc) and the core $\left(\mathrm{MgCO}_{3}\right)$. Images of lansoprazole and sucrose show they are located in the core of the drug. Figure $2 \mathrm{~d}$, an overlay of ion images, shows the complimentary distribution of these components in the cross-section. Two-dimensional chemical imaging of finished drug products can be invaluable for understanding the location and distribution of organic and inorganic constituents. It can also be critical in intellectual property cases where the patent law frequently dictates the location and function of materials. 
Analysis of precipitates/ inclusions in biological tissue can be problematic due to size/ quantity of the material available for analysis. Dissections often fail to yield sufficient quantities for Liquid Chromatography- Mass spectrometry. Direct analysis of cross-sectioned tissue specimens by TOF-SIMS is an option available due to its ability to generate a mass spectrum from only a few microns in size. Figure 3 shows an optical image of a rat kidney cross-section containing a precipitate ${ }^{2}$. The TOF-SIMS spectrum of the precipitate had an intense molecular ion at $\mathrm{m} / \mathrm{z} 331$, corresponding to the mass of the deestrification metabolite of the prodrug Xemiliofiban. Figure $3 \mathrm{~b}$ is a cumulative image of the ions related to the metabolite. There is good correlation of the TOF-SIMS metabolite image with the optical image of the precipitate.

[1] D. A. Cole et al, Conference Proceedings-6th World Biomaterials Congress, (2000).

[2] S. Levin et al, Toxicologic Pathology, 27 (1999), 38-43.
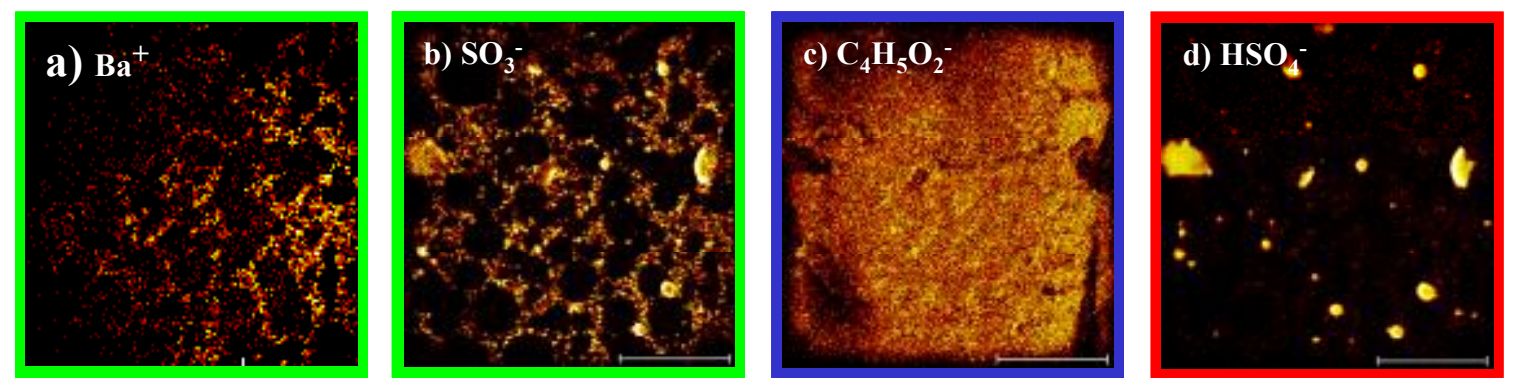

Figure 1. TOF-SIMS images of intense ions characteristic of a-b) $\mathrm{BaSO}_{4}$, c) PMMA components of Simplex-P cement, and d) Tobramycin sulfate. Scale bar $-100 \mu \mathrm{m}$.
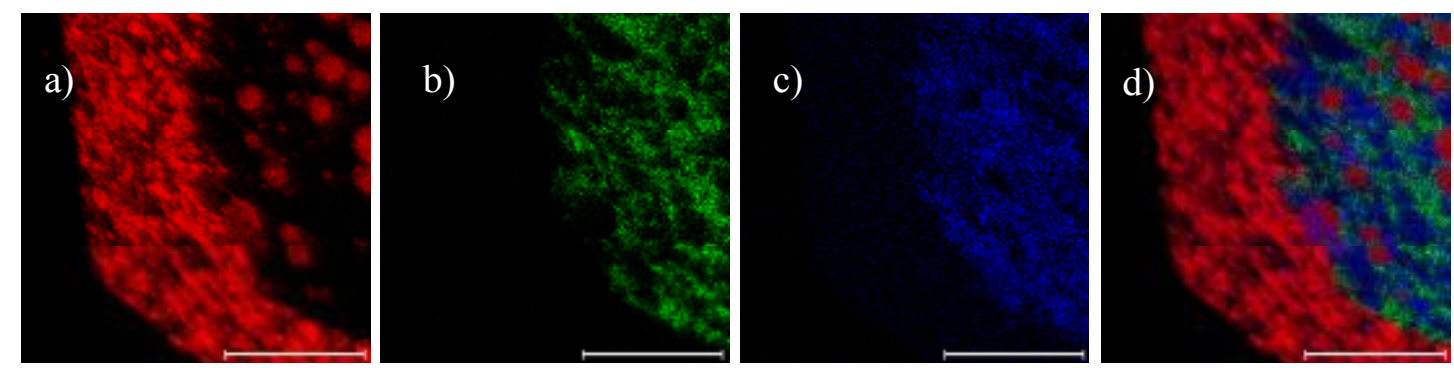

Figure 2. TOF SIMS image of a) Mg, b) Lansoprazole, c) Sucrose, d) Overlay of the three components. Scale bar $-100 \mu \mathrm{m}$.

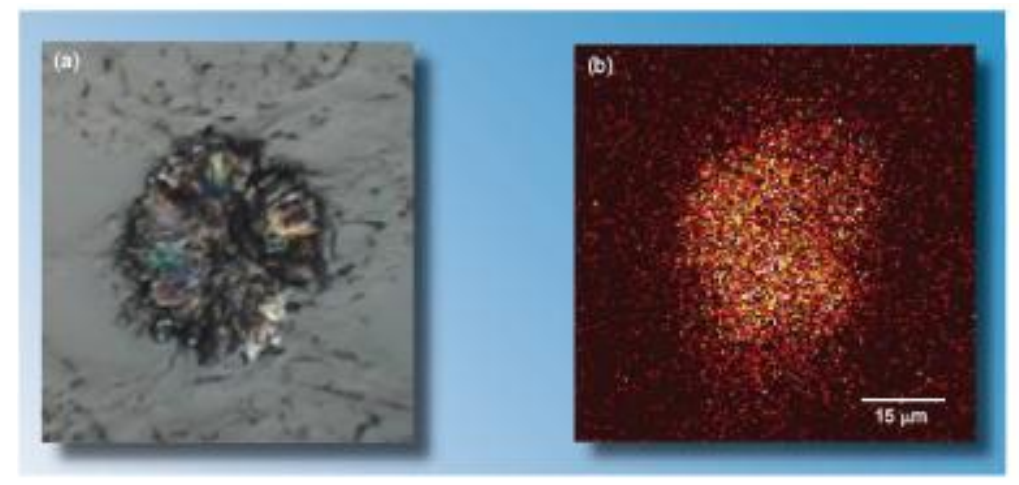

Figure 3. a) Optical image of the cross-sectioned precipitate and b) TOF-SIMS image of the precipitate using ten metabolite specific ions. 